\title{
OPTICAL COMMUNiCATION TERMinAl FOR MOBILE ROBOTS
}

\author{
Daria Stepanova \& Valentin Pryanichnikov
}
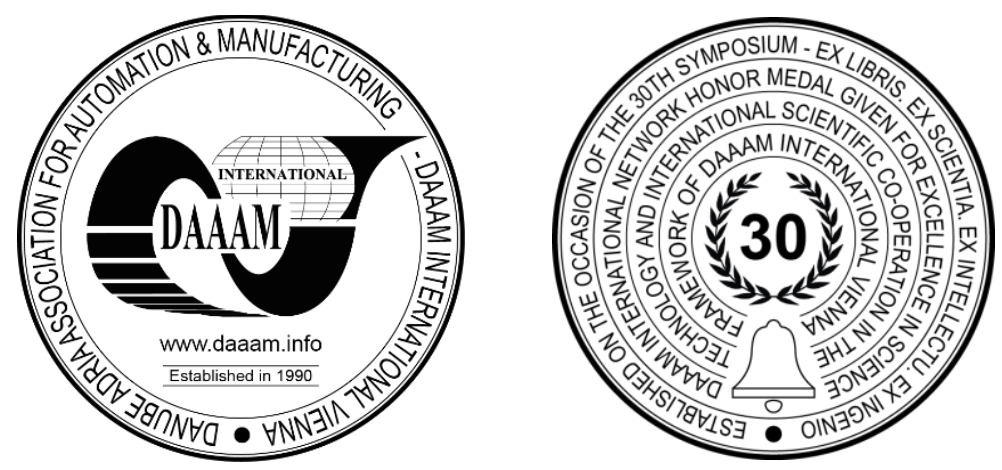

This Publication has to be referred as: Stepanova, D[aria] \& Pryanichnikov, V[alentin] (2020). Optical Communication Terminal For Mobile Robots, Proceedings of the 31st DAAAM International Symposium, pp.0334-0340, B. Katalinic (Ed.), Published by DAAAM International, ISBN 978-3-902734-29-7, ISSN 1726-9679, Vienna, Austria

DOI: $10.2507 / 31$ st.daaam.proceedings.047

\begin{abstract}
Optical wireless communication is the promising technology for mobile platforms high-speed data exchange. Technology has already demonstrated applications in different fields and for different platforms: from satellites to trains and unmanned aerial vehicles. The technology has several valuable advantages comparing to existing radio frequency solutions: communication terminal and antenna are smaller; connection is more secured and energy efficient. However, to achieve the mass, power and communication parameters optimal ratio it is required to provide the highly accurate laser beam pointing. This can be achieved using the visual system as a feedback and high-speed control loop. Even though there are several approaches for the laser terminal design for mobile platforms, development of an alternative high-frequency tracking system and its implementation using the visual feedback remains relevant for applications on mobile robotic systems. Current work is dedicated to study a gimbal-based optical terminal and pointing system for mobile robots communication. Based on the previously derived requirements for the optical terminal, a pointing and tracking system architecture is developed. The mathematical model of a two-axes gimbal including the mutual influence of the pointing system drives and platform is derived together with the model of control system. System stability and accuracy are evaluated considering several scenarios and parameters of the optical terminal are discussed.
\end{abstract}

Keywords: Pointing system; optical communications; mobile robots; gimbal system

\section{Introduction}

Precise pointing and tracking systems significantly increase performance of robotics communication devices, vision systems and other instruments for information gathering about the environment [1-5]. Simple visual systems use closeloop algorithms for optical spot center deviation to determine the misalignment vector and correct the angles of actuators rotation [6]. The use of such systems on mobile robots or unmanned aerial vehicles (UAVs) provides the advantages of reducing the delay in the control cycle and increasing the pointing accuracy, which is especially important for robots' operations under uncertainty and extreme conditions [7-10].

This work proposes the development approach of a new generation pointing system for the implementation of highprecision pointing and tracking. Distinctive features of the system consist of the improvement of an algorithm for optical spot center determination and the pointing system control cycle update rate acceleration through implementation of this algorithm on the FPGA. 
For further simulation, following scenarios involving mobile robots' dynamics are considered. Several robots move relative to each other at a certain distance and transmit information using a laser beam. To implement this configuration, it is necessary to ensure the pointing of the optical transmitter with an accuracy of the milliradian and to maintain the position throughout the entire communication session. For stable operation of the optical pointing system, it is necessary to develop an algorithm that can provide the smallest error in determining the center of the beam, taking into account the ratio of the noise level to the signal level.

\section{Optical terminal design requirements and architecture}

The set of requirements for the pointing system was derived earlier in [11] through the dynamic modelling and relative movement simulation of a group of mobile robots, a communication channel between them, energy balance and mass balance of robots. Several scenarios including different trajectories and optical system characteristic were proposed. Requirements including values of the maximum distances at which data transmission is possible and the necessary pointing accuracy for each considered scenario are visualized in the Table 1.

\begin{tabular}{|l|l|l|}
\hline Parameter & Value & Dimension \\
\hline Maximum distance between robots & Up to 10 & $\mathrm{~km}$ \\
\hline Relative view angles & 57 & $\mathrm{deg}$ \\
\hline Relative angular rates & $0.2-35.6$ & $\mathrm{deg} / \mathrm{s}$ \\
\hline Relative angular accelerations & Up to 2.8 & $\mathrm{deg} / \mathrm{s}^{2}$ \\
\hline Communication speed & Up to 2.1 & $\mathrm{Gbs}$ \\
\hline Energy consumption & Up to 22 & $\mathrm{~W}$ \\
\hline Pointing accuracy & 0.01 & $\mathrm{mrad}$ \\
\hline Optical terminal mass & 5 & $\mathrm{~kg}$ \\
\hline Optical terminal size & $0.2 \times 0.2 \times 0.4$ & $\mathrm{~m}$ \\
\hline
\end{tabular}

Table 1. Optical terminal requirements definition

Based on the derived requirements for the pointing system, an architecture of the optical terminal implementation using a gimbal-based suspension system with two degrees of freedom is proposed. The optical communication terminal includes a laser transmitter, a photodetector, a data transmission system and a beacon, a guidance system and an information processing system. The terminal structure is shown in Fig. 1.

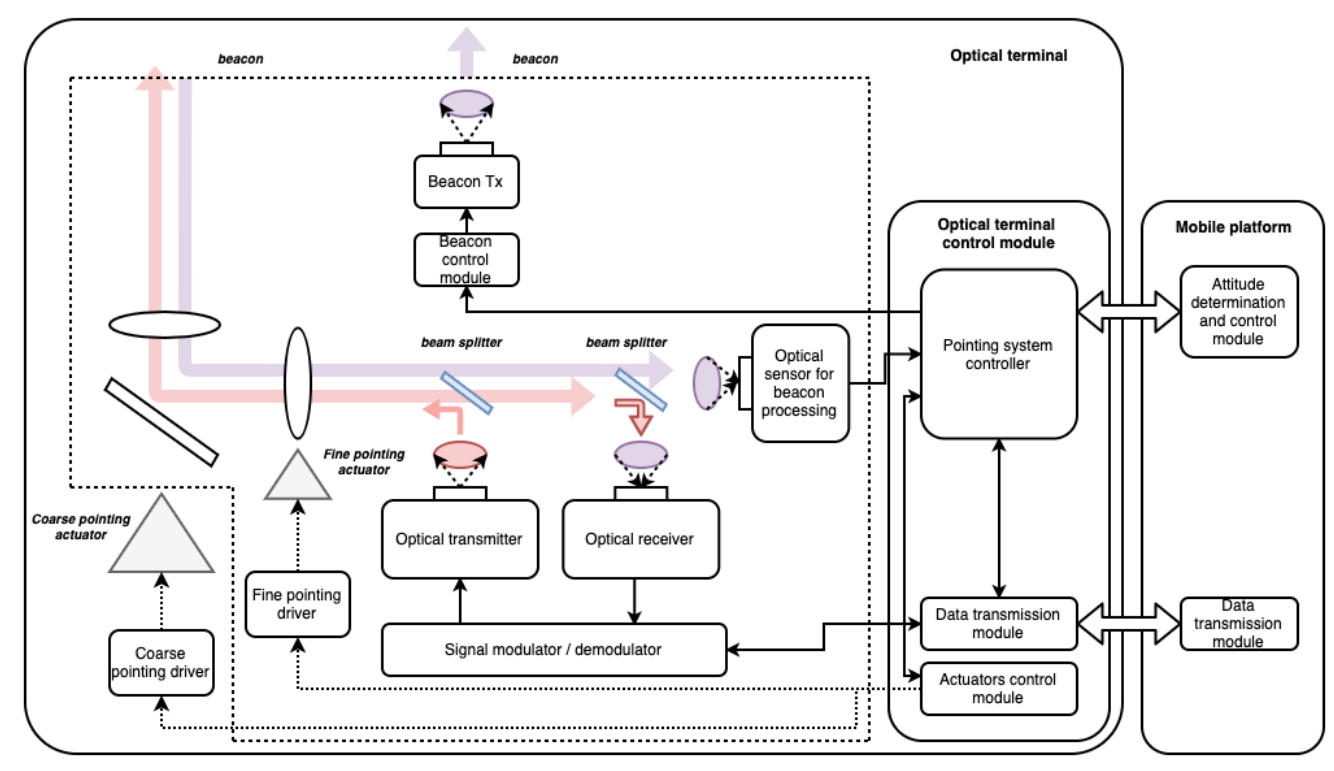

Fig. 1. Optical terminal architecture

The laser beacon from one platform enters the optical system and telescope of the pointing system, after primary processing it reaches the pointing system controller. The controller then extracts the deviation data and sends it to the host computer. The pointing system, based on the processed signal of the optical spot, corrects the gimbal angles of rotation, thereby establishing the propagation direction of the outgoing optical beam. The main computer sets the initial orientation angles for the control system and generates data for transmission. Angle encoders are used to set the feedback in the drive design, providing information about the absolute rotation of the drives. 
Based on the obtained data, the difference between the current and required position of the actuator is calculated. During the initial beam spot search, when information from the video sensor is not available, only data from the platform orientation and the position of the partner platform are used backed up with low speed radio channel. It is assumed that at each moment in time the communication platform estimates its orientation and position, and also has sufficient information about the location and velocity vector of the partner platform. Thus, each platform can estimate the vector and tracking angles based on the platform's initial position and speed.

The 3D model of the pointing system is based on a monoblock approach (Fig. 2). The support base ensures the installation of all the drive units of the terminal. The gimbal assembly consists of a base with structural elements, two drives including motors and resolvers, a telescope with a truncated aperture and collimators, electronic boards, optical and interface cables. The terminal drive axles are the supporting elements of the moving parts. They connect with the housings through the bearing units. Such installation of bearing assemblies provides the required accuracy of installation of the axes and moving parts of the drive.

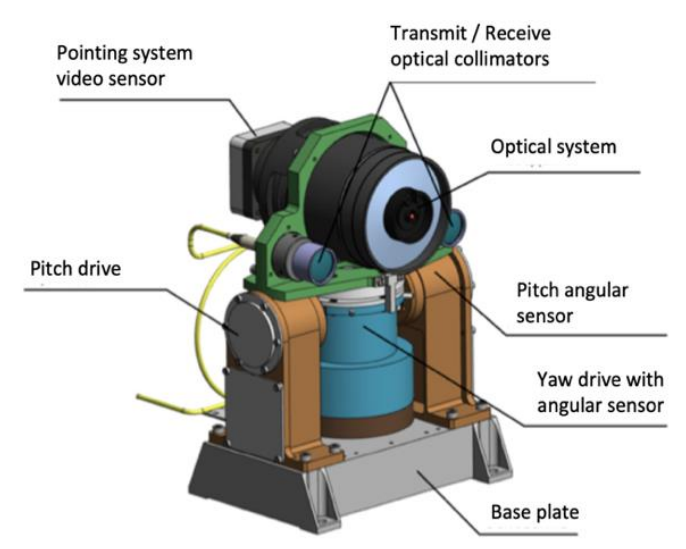

Fig. 2. Optical terminal 3D model

The pointing system is a two-axis drive carrying the laser communication collimators and the optical telescope. Pointing is achieved by changing two angular coordinates, elevation and azimuth (pitch and yaw), relative to the reference plane. The elevation motor and the elevation angle sensor are fixed on a mobile platform base and provide a tilt of the movable unit by $\pm 10^{\circ}$. The azimuth drive tilts with the movable unit by $\pm 30^{\circ}$. The pointing system electronics processes information from elevation and azimuth sensors, as well as images from a video camera, and based on these data forms control signal for the motors. The angle sensor board with CMOS sensor and FPGA is installed on the mobile module, the rest of the boards are at the base of the device. The terminal of the laser communication system is connected directly to the collimators using a pair of optical waveguides, bypassing the electronics unit of the guidance system.

\section{Development of a mathematical model and control system for the pointing system}

The model of a two-axis suspension gimbal is derived as a rigid body rotating around two axes passing through the laser beam diode (Fig. 3) In the course of work [12, 13] similar systems are described. However, they only discuss the symmetric body, while current approach is focused on the unsymmetrical body.

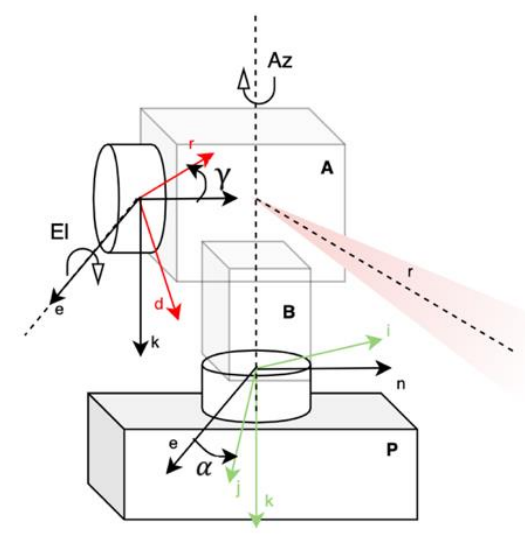

Fig. 3. Pointing system schematics 
The model uses following coordinate systems (CS): CS $\mathrm{P}_{\text {nek }}$ is connected to the platform on which the terminal is installed, $C S B_{i j k}$ is connected to the azimuth coarse pointing drive, the $A_{i e d} C S$ is connected to the elevation coarse pointing drive. The angles of rotation $\alpha$ and $\gamma$ are determined as follows: the drive B installed on the housing rotates the body of the pointing system around the axis k by the angle $\alpha$; drive A rotates the optical transmitter and receiver by an angle $\gamma$ around the e axis. Based on the law of angular momentum conservation the equation for the elevation angle is derived [14]:

$$
\begin{aligned}
& \mathbf{T}=\frac{d K_{A}}{d t}+\boldsymbol{\omega}_{A} \times \boldsymbol{K}_{A} \\
& T_{e l}=\dot{K}_{e}+\omega_{A d} K_{r}-\omega_{A r} K_{d} \\
& J_{A e} \omega_{A e}^{\dot{*}}=T_{e l}+\left(J_{A d}-J_{A r}\right) \omega_{A r} \omega_{A d}-J_{A r e}\left(\omega_{A r}^{\dot{*}}+\omega_{A e} \omega_{A d}\right)+J_{A r d}\left(\omega_{A r}{ }^{2}-\omega_{A d}{ }^{2}\right)-J_{A d e}\left(\omega_{A d}+\omega_{A e} \omega_{A r}\right)
\end{aligned}
$$

where $\boldsymbol{T}_{e l}, \boldsymbol{T}_{a z}$ - angular momentum of drives; $J_{A}, J_{B}$ - inertia characteristics of azimuth and elevation drives; $\boldsymbol{\omega}_{\boldsymbol{P}}, \boldsymbol{\omega}_{\boldsymbol{B}}$, $\boldsymbol{\omega}_{\boldsymbol{A}}$ - angular speeds in corresponding CS; $\boldsymbol{K}_{\boldsymbol{A}}$ - system kinetic moment relative to azimuth angle. Assuming that system does not include dynamic imbalance and coordinate system transformation:

$$
J_{A e} \dot{\omega}_{A e}=T_{e l}-\left(J_{A d}-J_{A r}\right) \omega_{A d}^{2} \tan (\gamma)+\frac{\omega_{B n} \omega_{A d}\left(J_{A d}-J_{A r}\right)}{|\cos (\gamma)|}
$$

Azimuth equation of motion is also derived from the law of angular momentum conservation [14, 15]:

$$
\begin{aligned}
& \omega_{B} \times H_{k}=\omega_{B n}\left(J_{B n e} \omega_{B n}+J_{B e} \omega_{B e}+J_{B k e} \omega_{B k}+J_{A r e} \omega_{A r}+J_{A e} \omega_{A e}+J_{A d e} \omega_{A d}\right)-\omega_{B e}\left(J_{B n e} \omega_{B n}+\right. \\
& \left.J_{B n e} \omega_{B e}+J_{B n k} \omega_{B k}\right)-\omega_{B e}\left(J_{A r} \omega_{A r}+J_{A r e} \omega_{A e}+J_{A r d} \omega_{A d}\right) \cos \gamma-\omega_{B e}\left(J_{A d} \omega_{A r}+J_{A d e} \omega_{A e}+J_{A d} \omega_{A d}\right) \operatorname{sin\gamma }
\end{aligned}
$$

After simplification:

$$
\begin{aligned}
J_{A e} \omega_{A d}^{\dot{a}}=T_{a z} \cos \gamma & -\frac{\omega_{B n} \omega_{B e}\left(J_{B k}\left(1+\sin ^{2} \gamma\right)+2 J_{A r} \sin ^{2} \gamma+\left(J_{B e}-J_{B n}\right) \cos ^{2} \gamma\right)}{\cos \gamma} \\
& -\omega_{A d} \omega_{A e}\left(J_{A r}+J_{B k}+\left(J_{A r}+J_{A d}\right) \cos ^{2} \gamma\right) \tan (\gamma) \\
& +\left(\omega_{P l}^{\cdot} \cos \alpha+\omega_{P J} \sin \alpha\right)\left(J_{A r}+J_{B k}\right) \sin \gamma-\omega_{B e} \omega_{P k}\left(J_{A r}+J_{B k}\right) \sin \gamma \\
& +\frac{\omega_{B n} \omega_{A e}\left(J_{A r}+J_{B k}-J_{A e} \cos ^{2} \gamma\right)}{\cos \gamma}+2 \omega_{A d} \omega_{B e}\left(J_{A r}+J_{B k}\right) \tan \gamma
\end{aligned}
$$

where $\dot{\omega}_{B}$ - angular speed change; $J_{k}$ - inertia moment around $k$ axes; $\boldsymbol{K}$ - total momentum of the system. The system of equation (4) and (6) describes the dynamic behaviour of the pointing system and includes the relative influence of drives on each other. The electrical drive motor model can be described as:

$$
\begin{aligned}
& u_{x}=R_{x} i_{x}+L_{x} \frac{d i_{x}}{d t}+k_{e x} \dot{\alpha} \\
& u_{y}=R_{y} i_{y}+L_{y} \frac{d i_{y}}{d t}+k_{e y} \dot{\gamma}
\end{aligned}
$$

where $u_{x}$ and $u_{y}$ - electrical motor voltage, $R_{x}$ and $R_{y}-$ stator resistances, $L_{y}$ and $L_{y}-$ motor inductances, $k_{e x}$ and $k_{e y}$ - back EMF coefficients. At this stage of the design, a PID controller is used to control the angles of the pointing system:

$$
G_{P I D}(s)=K_{P}+\frac{K_{i}}{s}+K_{D} s
$$

To close the tracking loop, it is also necessary to supplement the pointing system model with the module responsible for determining the deflection angle from the beacon signal and the resolver model. The transient function of the sensor is represented by the gain and delay link. The transient function of the resolver module is:

$$
G_{r e s}=\frac{\omega_{n}^{2}}{s^{2}+2 \zeta \omega_{n} s+\omega_{n}^{2}}
$$

where $\omega_{n}-$ natural frequency of resolvers, $\zeta-$ damping factor. 


\section{Modelling results}

Based on the described mathematical model, a Simulink model of pointing system was developed for the subsequent analysis. Fig. 4 visualizes this model. The gamma ref and alfa ref blocks define the direction of propagation of an optical beam. The centroid function calculates the deviation and feeds the tracking angle to the PID controllers. PID controllers then define the state of the motors and the position of the drives by generating torque. The control loop is closed by resolvers' feedback. The Platform block is responsible for vibration and movement of the mobile platform. In this model, the mobile platform introduces low-amplitude sinusoidal disturbances at a frequency of 100 to $300 \mathrm{~Hz}$ in accordance with the vibration characteristics of the motor drives. The values of the PID controller coefficients were obtained using the Ziegler-Nichols method [16].

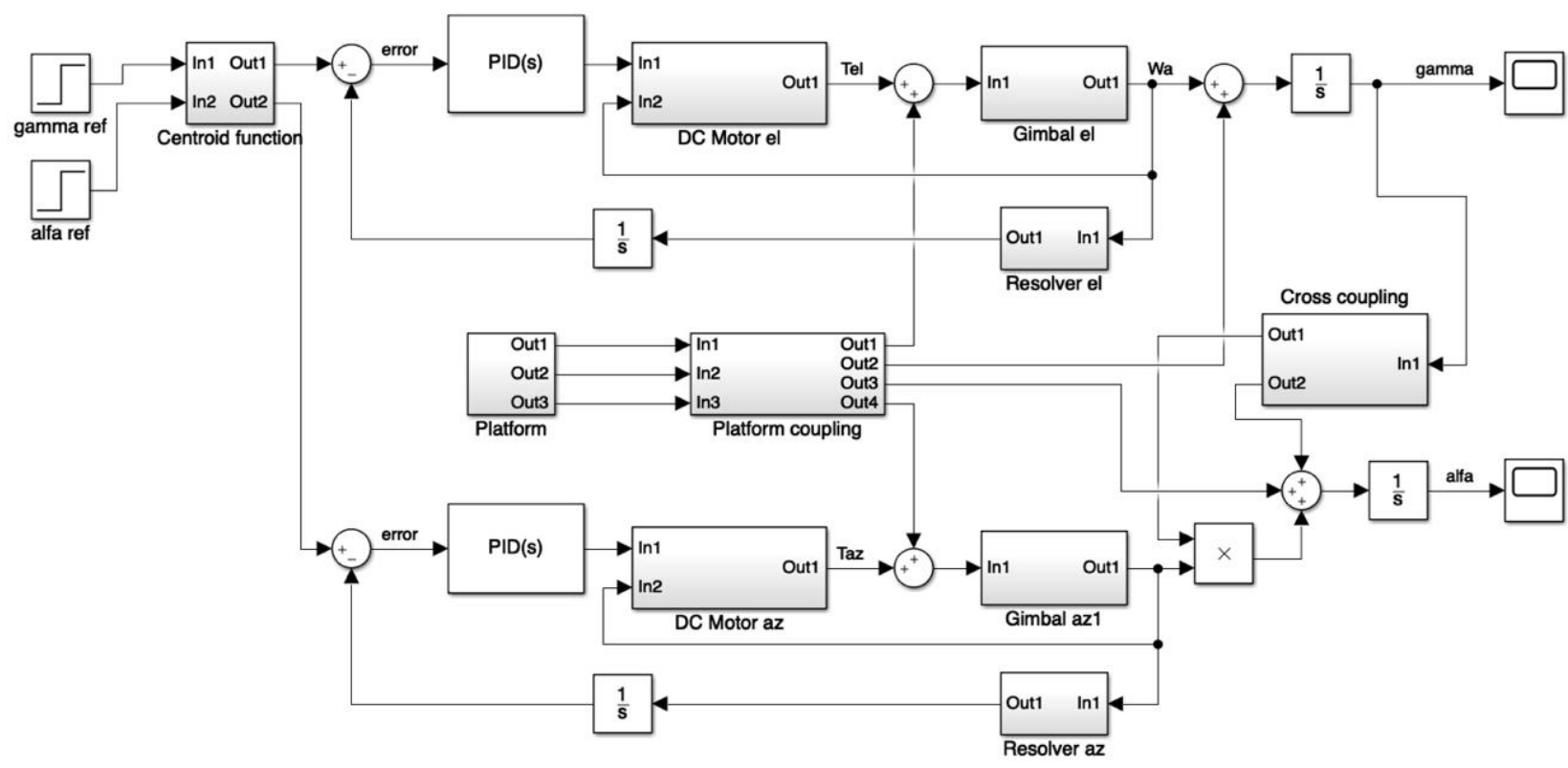

Fig. 4. Pointing system model in Simulink

The PID controller characteristics before and after tuning are shown in Fig. 5.

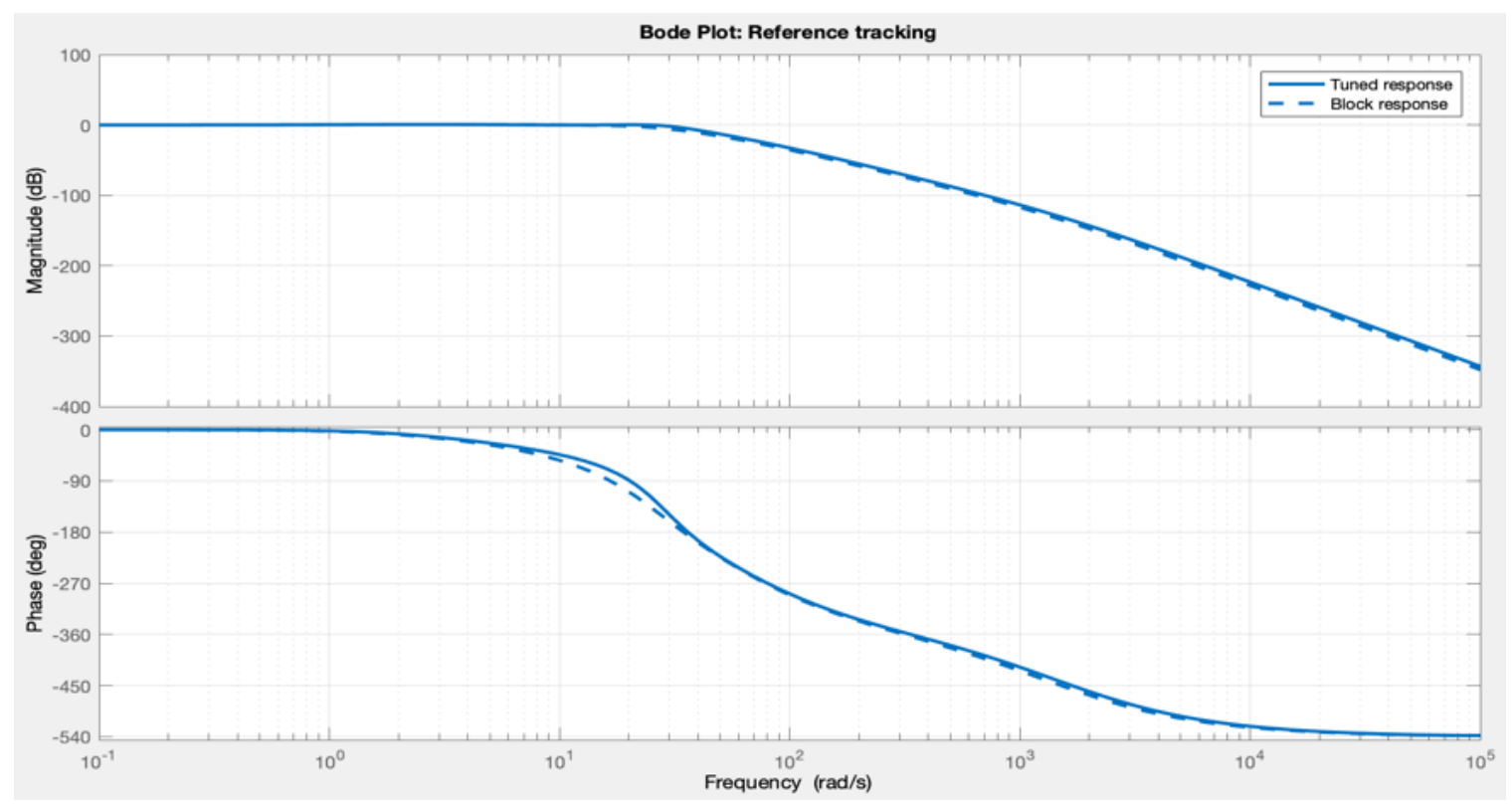

Fig. 5. PID controller characteristics before and after tuning

Step responses in two angles are visualized on Fig. 6. Settling time was decreased to 0.65 and 0.53 msec, overshoot does not exceed $9 \%$ and $5 \%$ for the alpha and gamma angles respectively. 

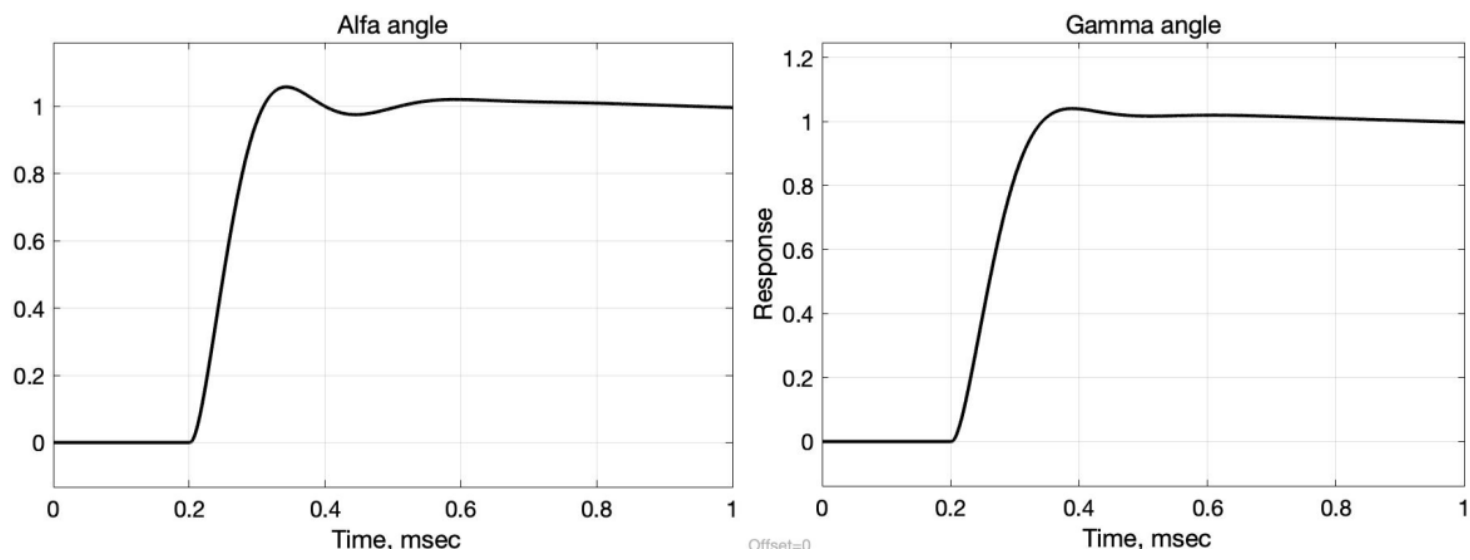

Fig. 6. System step response

The figures below present the system's reactions to influences caused by the mutual movement of mobile robots for several scenarios: Fig. 7 - movement of robots on straight parallel paths; Fig. 8 - movement of robots along a complex trajectory.
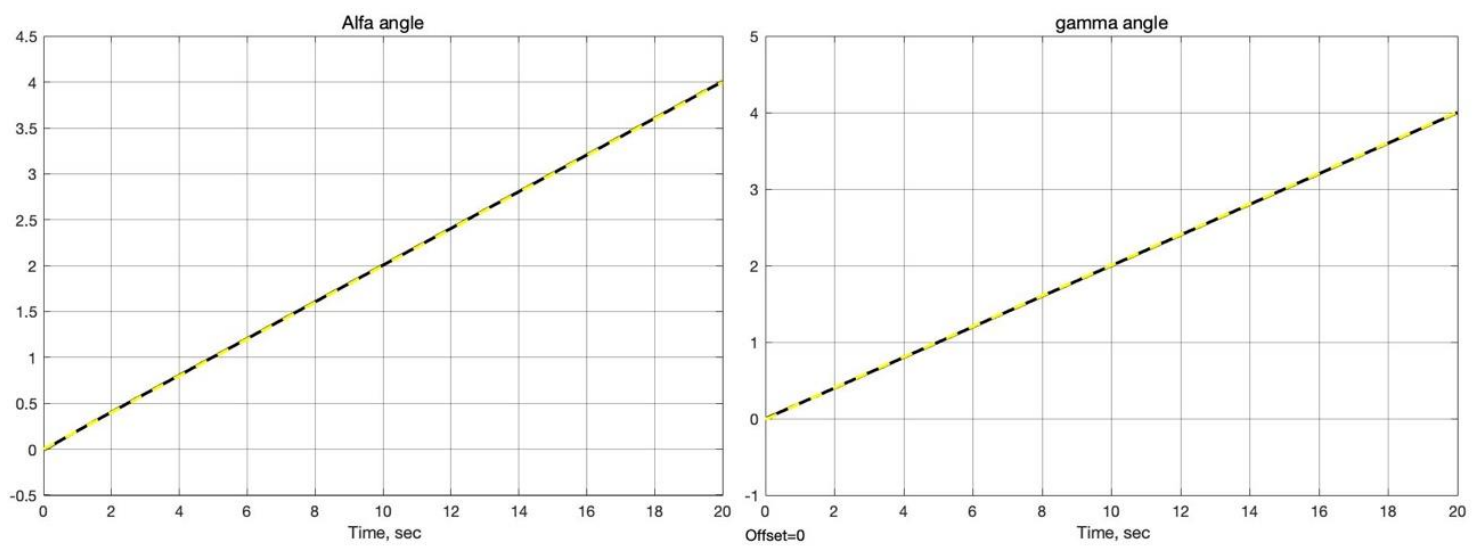

Fig. 7. The response of the system to a change in the guidance angle when mobile robots move along a straight path
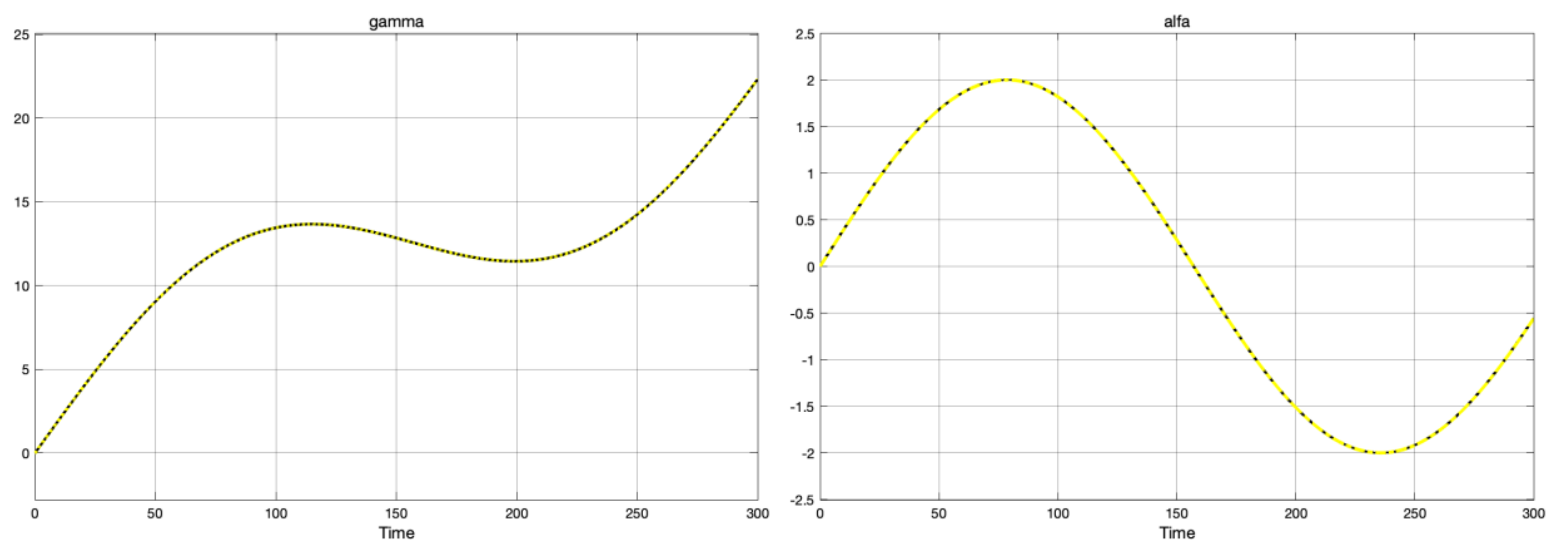

Fig. 8. The response of the system to a change in the guidance angle when moving mobile platforms relative to each other along a complex trajectory

The conducted simulation has demonstrated the feasibility of the proposed design approach. Mobile robot's movement modelling together with closed loop control system have proved that if the optical sensor is capable of detecting the centroid of laser spot misalignment with an accuracy 2"' the communication speed can reach 2 Gbps. For the future work, the developed model shall include the vibrational profile of gimbal system and robotic drives system and general error budget shall be conducted to evaluate the boundary conditions when the communication is still possible. 


\section{Conclusion}

The evolution of mobile robotic platforms in terms of data generation capabilities and data transmission requirements opens up new possibilities for existing and new technologies. The challenge of creating a compact optical communication terminal using a two-axis gimbal for installation on small mobile robot is studied in this paper. The characteristics of the pointing system impose restrictions on the data rate and the size of the optical terminal, so the design of the pointing system is key to the entire communications terminal design. In current work, a control system design is proposed and mathematical model of the overall optical terminal is simulated to verify the required communication speed and optical terminal characteristics.

As for future work, the optical terminal model shall be complemented with gear friction model and dynamic and static imbalances. It is also necessary to upgrade the model of the platform motion, taking into account the experimental data on the vibration background. In addition, the subsequent work shall include the controller implementation and real hardware tests. Regarding the architecture of the guidance system, it makes sense to supplement the model with a precision guidance system using piezo drives and develop a controller to adjust the four drives taking into account their mutual influence on each other.

\section{References}

[1] V. W. Chan, "Free-space optical communications," Journal of Light- wave technology, vol. 24, no. 12, pp. 47504762, 2006.

[2] T.-H. Ho, "Pointing, acquisition, and tracking systems for free-space optical communication links," Ph.D. dissertation, 2007.

[3] V. W. Chan, “Optical satellite networks,” Journal of Lightwave Technology, vol. 21, no. 11, p. $2811,2003$.

[4] Z. Sodnik, B. Furch, and H. Lutz, "Free-space laser communication activities in Europe: SILEX and beyond," in Lasers and Electro-Optics Society, 2006. LEOS 2006. 19th Annual Meeting of the IEEE, 2006, pp. 78-79.

[5] Keizo Nakagawa, Akio Yamamoto, "Preliminary design of Laser Utilizing Communications equipment (LUCE) installed on Optical inter-orbit communications engineering test satellite", National Space Development Agency of Japan (NASDA)

[6] N. Perlot, M. Knapek, D. Giggenbach, J. Horwath, M. Brechtelsbauer, Y. Takayama, T. Jono, "Results of the optical downlink experiment KIODO from OICETS satellite to optical ground station oberpfaffenhofen (OGS-OP)," in Proceedings of SPIE, vol. 6457, 2007

[7] 1. Stepanova, Daria, Valentin Pryanichnikov, Sergey Khandorin, Andrey Kulchitsky, and Alexey Kuznetsov. "Developing A Highly Accurate Pointing System for Free." Journal of Communications 14, no. 12 (2019).

[8] T. S. Rose, D. W. Rowen, S. LaLumondiere, N. I. Werner, R. Linares, A. Faler, J. Wicker, C. M. Coffman, G. A. Maul, D. H. Chien, A. Utter, R. P. Welle, and S. W. Janson, "Optical communications downlink from a 1.5U cubesat: OCSD program," Proc. SPIE 10610, 109100T (2019).

[9] A. Carrasco-Casado, H. Takenaka, D. Kolev, Y. Munemasa, H. Kunimori, K. Suzuki, T. Fuse, T. Kubo-oka, M. Akioka, Y. Koyama, and M. Toyoshima, "LEO-to-Ground Optical Communications using SOTA (Small Optical TrAnsponder) -Payload Verification Results and Experiments on Space Quantum Communications-," Acta Astronaut. 139, 377-384 (2017).

[10] S. W. Janson and R. P. Welle, "The NASA Optical Communication and Sensor Demonstration Program: An Update," in 28th Annual AIAA/USU Conference on Small Satellites (2014), paper SSC14-VI-1.

[11] Stepanova, D., Pryanichnikov, V., Khandorin, S., Kuznetsov, A. and Kulchitsky, A., 2018. High-Speed Image Processing Technique Implementation For Pointing And Tracking System Enabling Free-Space Optical Communications. Annals of DAAAM \& Proceedings, 29.

[12] Abdo, Maher, Ahmad Reza Vali, Alireza Toloei, and Mohammad Reza Arvan. "Research on the cross-coupling of a two axes gimbal system with dynamic unbalance." International Journal of advanced robotic systems 10 , no. 10 (2013): 357.

[13] Masten, M.K. (2008) Inertially stabilized platform for optical imaging systems. IEEE Control Systems Magazine, vol. 28, pp. 47-64.

[14] Golubev, U. F. (2000) Foundations of Theoretical Mechanics, Lomonosov Moscow State University, 5-211-042441, Moscow

[15] K. A. Pupkov, N.D. Egupov, A. I. Barkin, A. V. Zaitsev, S. V. Kanushkin et al. (2004) Methods of classical and modern theory of automatic control. Federal State Budgetary Educational Institution of Higher Education Bauman Moscow State Technical University, 5-7038-2192-4, Moscow.

[16] Åström, Karl Johan, and Tore Hägglund. "Revisiting the Ziegler-Nichols step response method for PID control." Journal of process control 14, no. 6 (2004): 635-650. 